\title{
Density, viscosity and electrical conductivity of alkanolammonium ionic liquids
}

\author{
André Pinkert, Keng L. Ang, Kenneth N. Marsh*, and Shusheng Pang
}

ELECTRONIC SUPPLEMENTARY INFORMATION

Table 1 Decomposition temperatures of alkanolammonium ILs ${ }^{1}$ at a fractional mass loss of 0.1 at two different heating rates $\dot{\mathrm{T}}_{1 \mathrm{~K}}=1 \mathrm{~K} \cdot \mathrm{min}^{-1}$ and $\dot{\mathrm{T}}_{10 \mathrm{~K}}=10 \mathrm{~K} \cdot \mathrm{min}^{-1}$

\begin{tabular}{lccccc}
\hline & $\begin{array}{c}{[\mathrm{DAA}]} \\
\mathrm{t}_{1 \mathrm{~K}} /{ }^{\circ} \mathrm{C}, \mathrm{t}_{10 \mathrm{~K}} /{ }^{\circ} \mathrm{C}\end{array}$ & $\begin{array}{c}{[\mathrm{HEA}]} \\
\mathrm{t}_{1 \mathrm{~K}} /{ }^{\circ} \mathrm{C}, \mathrm{t}_{10 \mathrm{~K}} /{ }^{\circ} \mathrm{C}\end{array}$ & $\begin{array}{c}{[\mathrm{HPA}]} \\
\mathrm{t}_{1 \mathrm{~K}} /{ }^{\circ} \mathrm{C}, \mathrm{t}_{10 \mathrm{~K}} /{ }^{\circ} \mathrm{C}\end{array}$ & $\begin{array}{c}{[\mathrm{DEA}]} \\
\mathrm{t}_{1 \mathrm{~K}} /{ }^{\circ} \mathrm{C}, \mathrm{t}_{10 \mathrm{~K}} /{ }^{\circ} \mathrm{C}\end{array}$ & $\begin{array}{c}{[\mathrm{TEA}]} \\
\mathrm{t}_{1 \mathrm{~K}} /{ }^{\circ} \mathrm{C}, \mathrm{t}_{10 \mathrm{~K}} /{ }^{\circ} \mathrm{C}\end{array}$ \\
\hline $\mathrm{Fmt}$ & 50,90 & 63,111 & 100,151 & 120,158 & 110,154 \\
$\mathrm{Ac}$ & 38,62 & 95,132 & 100,142 & 106,144 & 84,119 \\
$\mathrm{Mal}$ & 61,98 & 108,136 & 108,141 & 97,142 & 106,136 \\
\hline
\end{tabular}

\section{References}

1 A. Pinkert, K. N. Marsh and S. Pang, Ind. Eng. Chem. Res., 2010, DOI: 10.1021/ie101250v. 